\title{
Existence and hardness of conveyor belts
}

\author{
Molly Baird \\ Department of Applied Mathematics \\ University of Washington \\ Seattle, WA, U.S.A. \\ mollycbaird@gmail.com
}

\author{
Sara C. Billey* \\ Department of Mathematics \\ University of Washington \\ Seattle, WA, U.S.A. \\ billey@uw.edu
}

\author{
Erik D. Demaine \\ MIT CSAIL \\ Cambridge, MA, U.S.A. \\ edemaine@mit.edu \\ Martin L. Demaine \\ MIT CSAIL \\ Cambridge, MA, U.S.A. \\ mdemaine@mit.edu
}

\author{
David Eppstein ${ }^{\dagger}$ \\ Computer Science Department \\ University of California \\ Irvine, CA, U.S.A. \\ eppstein@uci.edu
}

\author{
Sándor Fekete \\ Department of Computer Science \\ TU Braunschweig \\ Braunschweig, Germany \\ s.fekete@tu-bs.de
}

\author{
Graham Gordon \\ Department of Mathematics \\ University of Washington \\ Seattle, WA, U.S.A. \\ grahamwalkergordon@gmail.com
}

Sean Griffin

Department of Mathematics

University of California, San Diego

San Diego, CA, U.S.A.

griff.sean.t@gmail.com

\author{
Joseph S.B. Mitchell ${ }^{\ddagger}$ \\ Department of Applied Mathematics and Statistics \\ Stony Brook University \\ Stony Brook, NY, U.S.A. \\ joseph.mitchell@stonybrook.edu
}

\author{
Joshua P. Swanson \\ Department of Mathematics \\ University of California, San Diego \\ San Diego, CA, U.S.A. \\ jswanson@ucsd.edu
}

Submitted: Aug 14, 2020; Accepted: Oct 21, 2020; Published: Oct 30, 2020

(C) The authors. Released under the CC BY license (International 4.0). 


\begin{abstract}
An open problem of Manuel Abellanas asks whether every set of disjoint closed unit disks in the plane can be connected by a conveyor belt, which means a tight simple closed curve that touches the boundary of each disk, possibly multiple times. We prove three main results:

1. For unit disks whose centers are both $x$-monotone and $y$-monotone, or whose centers have $x$-coordinates that differ by at least two units, a conveyor belt always exists and can be found efficiently.

2. It is NP-complete to determine whether disks of arbitrary radii have a conveyor belt, and it remains NP-complete when we constrain the belt to touch disks exactly once.

3. Any disjoint set of $n$ disks of arbitrary radii can be augmented by $O(n)$ "guide" disks so that the augmented system has a conveyor belt touching each disk exactly once, answering a conjecture of Demaine, Demaine, and Palop.
\end{abstract}

Mathematics Subject Classifications: 52C26

\title{
1 Introduction
}

In 2001 (later published in $[1,2]$ ), Manuel Abellanas posed the problem of characterizing when a finite collection of closed disks in the plane can be spanned by a conveyor belt, and, in particular asked if every collection of disjoint unit disks admits a spanning conveyor belt. A conveyor belt for such a collection of disks is a continuously differentiable simple closed curve that touches the boundary of each disk at least once, is disjoint from the disk interiors, and consists of arcs of the disks and bitangents between them. We may imagine such a curve as made out of an elastic band wrapped tightly around the disks. Consequently, all the disks and the conveyor belt with them can turn without slipping. Two disks have the same orientation if they turn in the same direction when the conveyor belt is pulled, or equivalently if they are both on the same side of the belt. See Figure 1.

We review some of the history of Abellanas' question. Tejel and García found an example of disks with different radii that have no conveyor belt [9]. Abellanas then refined his conjecture as follows.

Problem 1 (Abellanas). Does every finite set of disjoint closed unit disks in the plane have a conveyor belt?

O'Rourke [14] independently considered a relaxation of the problem by allowing the curve to cross itself, with prescribed disk orientations and arbitrary radii. For his variant of the problem, not every system of disjoint unit disks has a conveyor belt, but for a belt

\footnotetext{
*Partially supported by the University of Washington Graduate School and the National Science Foundation grant DMS-1764012

${ }^{\dagger}$ Partially supported by the National Science Foundation grant CCF-1618301 and CCF-1616248

${ }^{\ddagger}$ Partially supported by the National Science Foundation (CCF-1540890, CCF-2007275), the US-Israel Binational Science Foundation (project 2016116), and DARPA (Lagrange).
} 


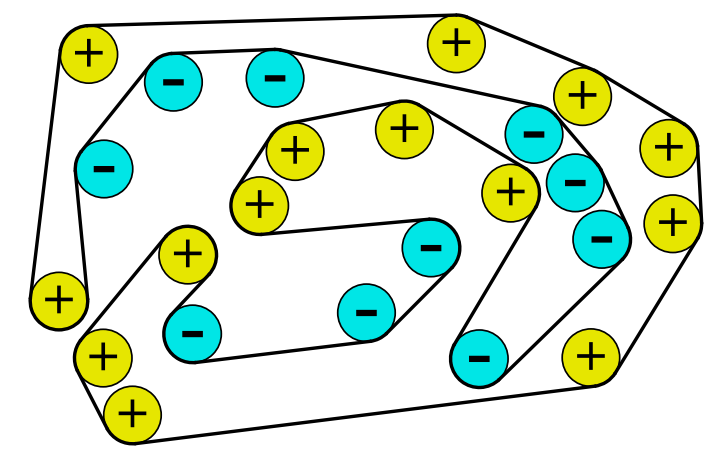

Figure 1: A conveyor belt on 24 nonoverlapping unit disks. The colors and markings of the disks indicate their orientations.

of this type to exist it is sufficient for a certain hull-visibility graph of the disks to be connected or for the disks to remain disjoint when expanded by a sufficiently large factor. Demaine, Demaine, and Palop [8,9] designed puzzle fonts, specified by a system of disks per character, with a unique conveyor belt in the shape of that character. If the conveyor belt is not shown, decoding the font becomes a puzzle for the viewer.

In this paper, we prove several related results on conveyor belts:

1. For unit disks whose sorted orders by $x$ - and by $y$-coordinates of their centers are the same (i.e., $x y$-monotone), there always exists a conveyor belt (Theorem 5), and a solution belt can be constructed in linear time after sorting (Theorem 6). The same method also applies to unit disks whose $x$-coordinates differ by two or more units and, more generally, to monotonically separated configurations (Definition 3).

2. Strengthening the known result that not every system of disks with arbitrary radii has a conveyor belt [9], we show that the decision problem is NP-complete for disks with arbitrary radii (Theorem 11).

3. A variation of the conveyor belt problem in which we constrain the belt to touch each disk exactly once (and disks may still have arbitrary radii) is also NP-complete (Theorem 8).

4. Both versions can be made to have a conveyor belt solution by adding $O(n)$ "small guide disks", answering a conjecture of Demaine, Demaine, and Palop [9] (Theorem 12). Conversely, $\Omega(n)$ guide disks are sometimes necessary (Theorem 14).

\section{Preliminaries}

Between any two disks $D_{1}$ and $D_{2}$, there are four bitangent line segments, as depicted in Figure 2. In the case when the two disks $D_{1}$ and $D_{2}$ have centers $\left(x_{1}, y_{1}\right)$ and $\left(x_{2}, y_{2}\right)$ with $x_{1}<x_{2}$, define the lower bitangent to be the bitangent which stays entirely below the 
line through the centers of $D_{1}$ and $D_{2}$. The upper bitangent is defined similarly. Define the inner bitangents as the two bitangents that cross the line between the centers of $D_{1}$ and $D_{2}$.

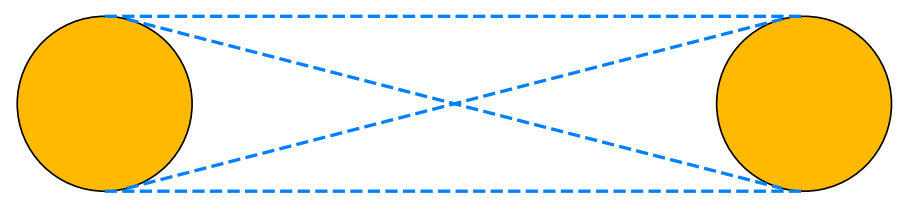

Figure 2: The four possible bitangents between two disks.

A conveyor belt for a collection of disks can be specified as follows. Between any two disks, the belt may travel along one of four bitangent line segments. The two disks have the same orientation if the bitangent does not pass through the line connecting their centers and they have different orientations otherwise. We call a bitangent unblocked if it does not intersect any disk, except at its endpoints. A conveyor belt can be completely specified by the cyclic order in which it visits each disk, together with the subset of disks lying inside the conveyor belt.

As a warm-up, the following family of disk configurations have an easy-to-construct conveyor belt. In this case, the disks in fact have a conveyor belt that touches each of them exactly once, along an arc of nonzero length.

Example 2. If the disks are in "general position" in the sense that no line intersects three disks, then there is always a conveyor belt for the collection. To construct a belt, let $P$ be any simple polygon with the disk centers as vertices; one often refers to such a $P$ as a (simple) polygonalization of the center points. Such a polygon $P$ must exist; a traveling salesman tour of the center points is one such (simple) polygonalization. Another approach is to choose one of the vertices of the convex hull of the circle centers and connect the remaining circle centers into a path in radial sorted order around the chosen vertex. Either cyclic order of the vertices of $P$ determines the order the belt touches the disks. To determine the orientations of the vertices, travel along $P$ cyclically and declare all disks where we turn left to be of one orientation and all disks where we turn right to be of the other orientation.

\section{Monotonically Separated Unit Disk Configurations}

We begin by fixing some notation and vocabulary. Suppose we have a sequence of $n$ disks with centers $\left(x_{i}, y_{i}\right)$ for $1 \leqslant i \leqslant n$. By rotating and relabeling the disks if necessary, we can assume without loss of generality that $x_{1}<x_{2}<\cdots<x_{n}$, so the disks are sorted by their $x$-coordinates.

Definition 3. We say that a sequence of disks is monotonically separated if $x_{1}<x_{2}<$ $\cdots<x_{n}$ and for every $i<j<k$, the $k$ th disk is disjoint from the convex hull of the $i$ th and $j$ th disks, and the $i$ th disk is disjoint from the convex hull of the $j$ th and $k$ th disks. 

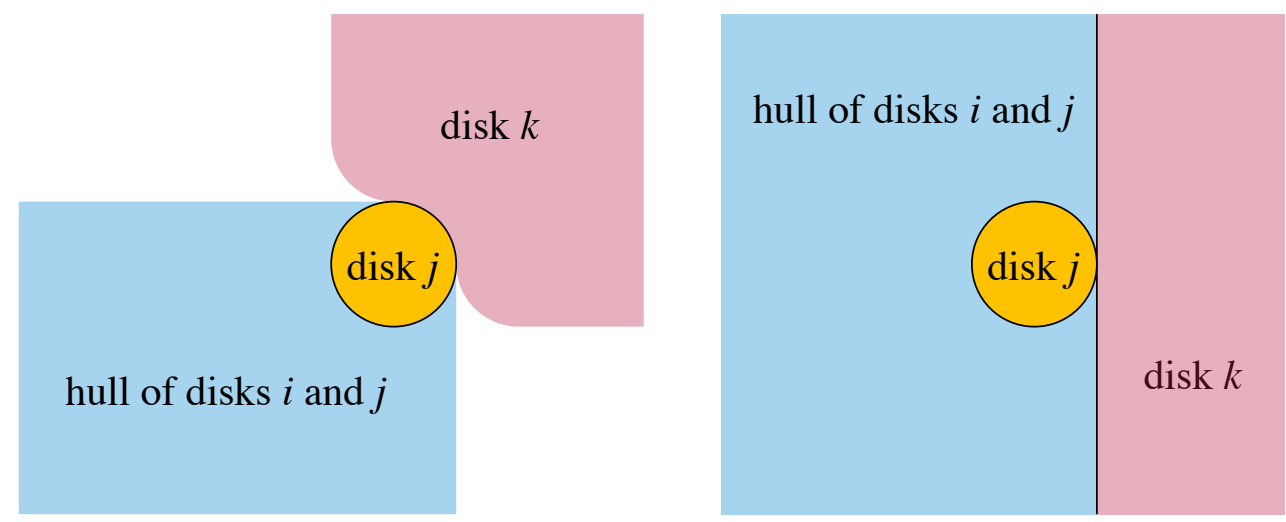

Figure 3: Diagrams of monotonically separated sequences. For unit disks with indices $i<j<k$ and with centers that are $x$ - and $y$-monotone (left) or whose $x$-coordinates differ by at least two units (right), the convex hull of disks $i$ and $j$ is confined to a disjoint region from disk $k$.

Lemma 4. Given a sequence of $n$ unit disks with $x_{1}<\cdots<x_{n}$, the disks are monotonically separated in either of the following two cases:

1. (xy-monotone) $y_{1}<\cdots<y_{n}$, so the centers are both $x$-monotone and $y$-monotone; or

2. (x-separated) for all $1 \leqslant i<n$, we have $x_{i+1}-x_{i} \geqslant 2$.

Proof. In the case where the $x$-coordinates differ by at least two units, the vertical line $x=x_{j}+1$ separates the convex hull of disks $i$ and $j$ from disk $k$. See Figure 3 . The other cases are similar.

We now come to one of our main results.

Theorem 5. Every monotonically separated sequence of unit disks has a conveyor belt.

Since the proof is lengthy, we briefly summarize the argument first. We start the construction with the convex hull of the disks, but since that may not touch disks on its interior, we use only the "upper half" of the convex hull as a segment of the final conveyor belt. We must then carefully thread the "lower half" of the belt through the disks to ensure each is touched without allowing the belt to intersect itself. We do so using certain "winding" and "unwinding" processes which allow us to iteratively extend partial lower belts indefinitely. The final belt is the concatenation of the upper and lower halves, which may well touch an individual disk multiple times, though it will have no self-intersections or self-overlaps.

Proof. Consider the upper convex hull of the disks, which is the part of the boundary of the convex hull passing clockwise from the leftmost point of the leftmost disk $L$ to the rightmost point of the rightmost disk $R$, not including these endpoints. Let $U_{1}, U_{2}, \ldots$ be the disks that contact the upper convex hull in between $L$ and $R$, referred to as the 
upper hull disks and listed with increasing $x$-coordinate. Let $D_{0}=L, D_{1}, D_{2}, \ldots, D_{k}=R$ be the disks that are not in the subsequence of upper hull disks, listed with increasing $x$-coordinate. We assume $k \geqslant 3$ since otherwise the boundary of the convex hull of the disks is a conveyor belt. We give an algorithm to find a conveyor belt which first traverses the convex hull above the upper hull disks and then weaves its way through the lower hull disks.

We begin by describing a key subroutine and some of its properties. We will shortly use this subroutine to iteratively construct a conveyor belt on the given disks. Given two consecutive disks $D_{i}$ and $D_{i+1}$, the "winding process" constructs two partial conveyor belts from $D_{i}$ to $D_{i+1}$ as follows. First, place a ray along the lower bitangent between $D_{i}$ and $D_{i+1}$ with the ray's vertex on $D_{i}$ and pointing the ray initially towards $D_{i+1}$. We report the partial conveyor belt from $D_{i}$ through the vertex of the ray to the point of tangency on $D_{i+1}$ as the "lower" of the two partial conveyor belts between $D_{i}$ and $D_{i+1}$. Second, starting with the ray along the "lower" bitangent between $D_{i}$ and $D_{i+1}$, continuously rotate the ray counterclockwise so that it stays tangent to $D_{i}$ at its vertex. As we rotate, we may shift the vertex of the ray to disks other than $D_{i}$ according to the following possible events considered in order.

1. The rotating ray may align with a tangent to $D_{i+1}$ for the first time. For example, this happens initially in the winding process when a lower tangent is found, but in the unwinding process below it will occur later and could be an inner tangent or an upper tangent between two disks that are not in the upper hull. In this case, we report the partial conveyor belt from $D_{i}$ through the vertex of the ray to this point of tangency as the "lower" of the two partial conveyor belts between $D_{i}$ and $D_{i+1}$. Continue rotating the ray in the same direction keeping the vertex on $D_{i}$.

2. The rotating ray may align with a tangent to some upper hull disk $U$ that lies between $D_{i}$ and $D_{i+1}$ in the $x$-sorted order of disks. If there is more than one such $U$, choose the one with the smallest $x$-coordinate. In this case, add to the current partial conveyor belt the bitangent along the ray between the disk the ray is currently rotating around and $U$. Shift the vertex of the rotating ray to $U$ and continue rotating the vertex of the ray counterclockwise around $U$ and inscribing an arc along the boundary of $U$.

3. The rotating ray may align with a tangent to $D_{i+1}$ for the second time. In this case, report a partial conveyor belt from $D_{i}$ to $D_{i+1}$ as before, this time calling it the "upper" partial conveyor belt. Stop the winding process.

See Figure 4 for the first partial conveyor belt, and see Figure 5 for an "animation" of the winding process.

We claim the winding process has the following properties.

(a) Both partial conveyor belts lie entirely within the convex hull of $D_{i}$ and $D_{i+1}$. 


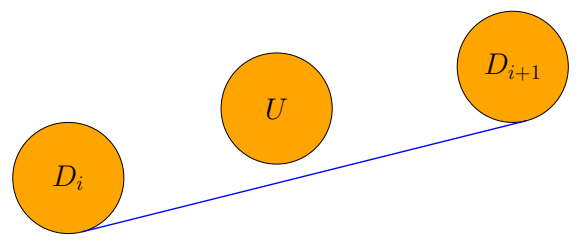

Figure 4: The first partial conveyor belt between $D_{i}$ and $D_{i+1}$ produced by the winding process, where $U$ is a disk on the upper hull.

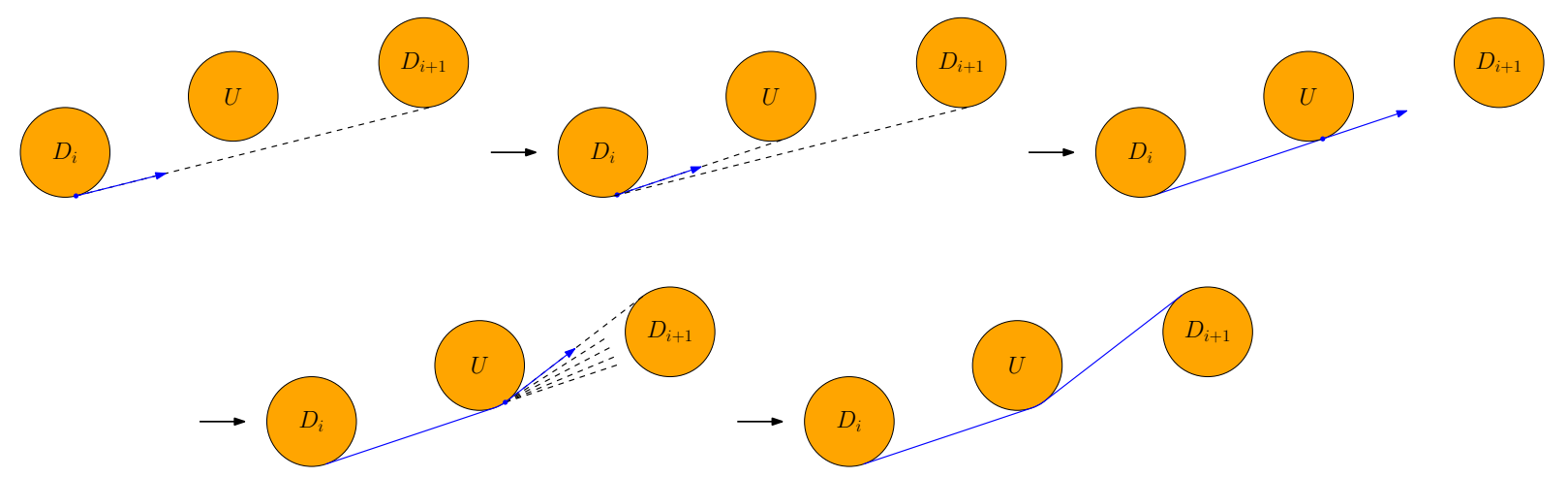

Figure 5: An "animation" of the second part of the winding process, which produces a second partial conveyor belt in the convex hull of $D_{i}$ and $D_{i+1}$.

(b) Both belts are disjoint from the upper convex hull, even though some upper hull disks may be touched by either belt.

(c) Both belts are valid partial conveyor belts touching $D_{i}$ and $D_{i+1}$ in the sense that they consist of arcs of disks and bitangents between them whose union is a continuously differentiable curve without self-intersection that is disjoint from all disk interiors.

Property (a) is easy to see, and (b) follows from (a). For (c), every property is clear except possibly that the partial belts may not be disjoint from all disk interiors. The ray initially points along the "lower" bitangent between $D_{i}$ and $D_{i+1}$, which cannot intersect the interior of an upper hull disk by definition of the upper hull so it is reported as part of the lower belt. As the winding process continues, the upper belt remains disjoint from all upper hull disk interiors with center $x$-coordinate between $D_{i}$ and $D_{i+1}$ by construction. The remaining disks $D_{j}$ are disjoint from the convex hull of $D_{i}$ and $D_{i+1}$ since the disks are monotonically separated by hypothesis, so both belts are disjoint from these remaining disks by (a), so property (c) holds.

The "unwinding process" is a slight variation on the winding process. Initially, the ray is placed exactly as before along the lower bitangent from $D_{i}$ to $D_{i+1}$ except its direction is reversed, with the vertex still at $D_{i}$. The vertex rotates counterclockwise, continuing exactly as before. The belts created by the unwinding process also satisfy (a)-(c) above by virtually the same reasoning. See Figure 6 for an example of the unwinding process, which produces two partial conveyor belts. 


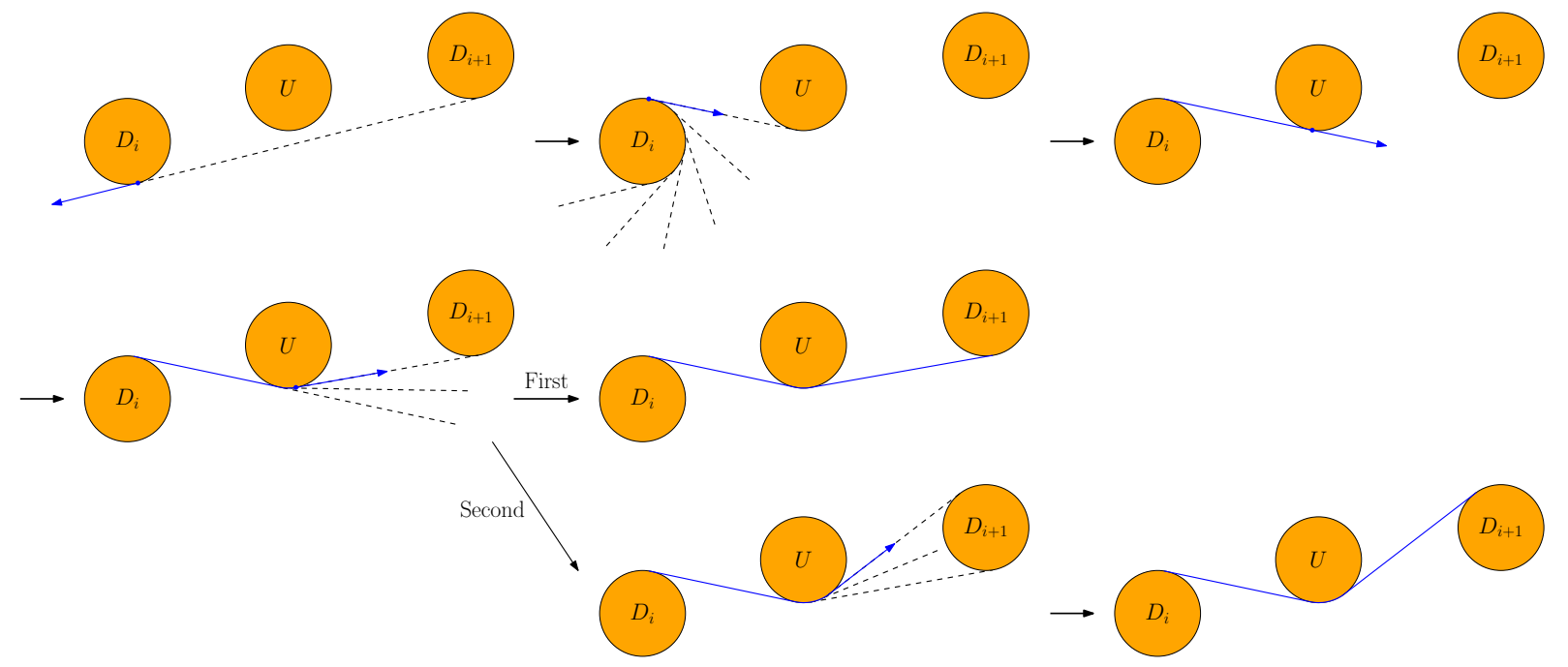

Figure 6: An "animation" of the unwinding process, which produces two partial conveyor belts between $D_{i}$ and $D_{i+1}$.

Now consider any triple of disks $D_{i-1}, D_{i}, D_{i+1}$. Suppose for simplicity that the centers of $D_{i-1}, D_{i}, D_{i+1}$ are not collinear. The boundary of the convex hull of $D_{i-1}$ and $D_{i}$ intersects the boundary of $D_{i}$ in a closed semicircle, and the boundary of the convex hull of $D_{i}$ and $D_{i+1}$ intersects the boundary of $D_{i}$ in another closed semicircle. Let $\alpha$ denote the intersection of these two semicircles. Then $\alpha$ is a closed arc on the boundary of $D_{i}$. We may now paste together two of the four partial belts from $D_{i-1}$ to $D_{i}$, an arc containing $\alpha$, and two of the four partial belts from $D_{i}$ to $D_{i+1}$ as in Figure 7, resulting in four partial belts from $D_{i-1}$ to $D_{i+1}$. Since the disks are monotonically separated, one may check that property (a) ensures each resulting partial belt from $D_{i-1}$ to $D_{i+1}$ has no self-intersections and continues to satisfy (b) and (c).
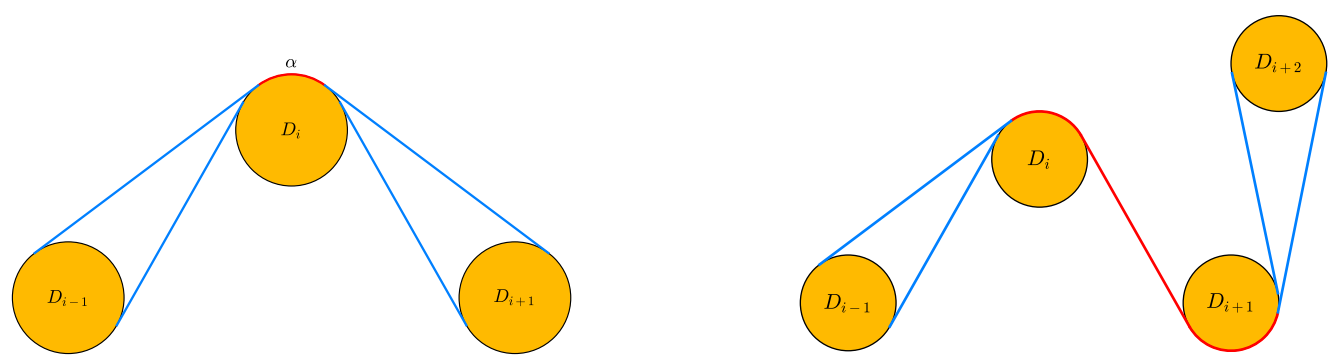

Figure 7: The first picture shows the four partial conveyor belts between three adjacent lower disks. The red arc labeled $\alpha$ is required to be part of the final belt. The second picture shows the next iteration of the algorithm after extending the four possible belts to include $D_{i+2}$. Again the red part of the belt is required in any further growth of the belt, and the blue forks indicate the four optional extensions. 
The full algorithm proceeds as follows. Begin with four partial conveyor belts between two adjacent lower disks. Iteratively extend these belts as above to produce four partial conveyor belts between ever larger sets of adjacent lower disks satisfying (b) and (c). For example, see Figure 7. Ultimately, this will create four partial conveyor belts from $L$ to $R$. Of these four, choose the one whose tangents are on the lower sides of $L$ and $R$. Now glue that partial conveyor belt to the upper hull by way of arcs along $L$ and $R$ to create a valid conveyor belt touching each disk at least once.
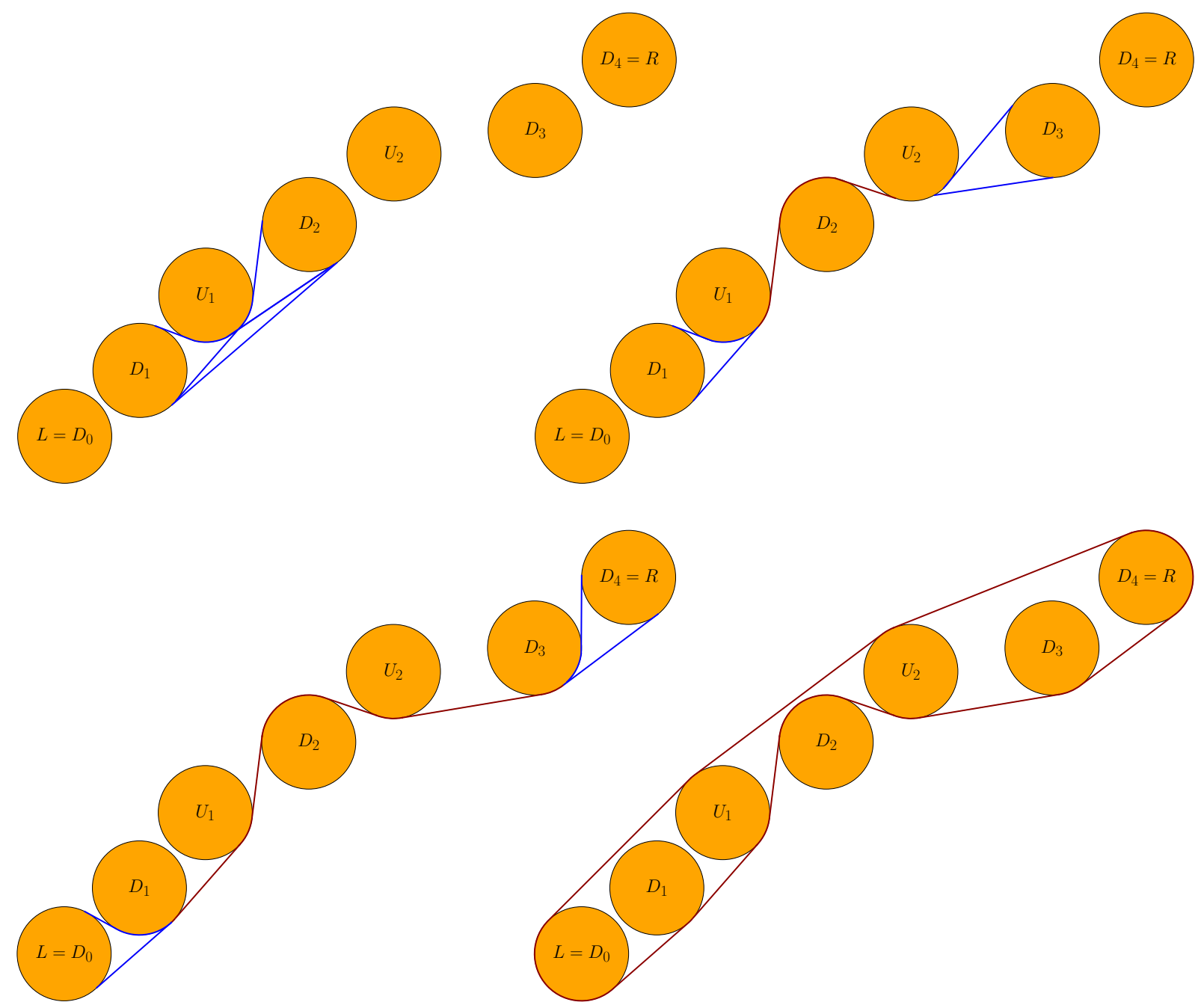

Figure 8: A sequence of $x$ - and $y$-monotone unit disks and several stages of our algorithm. The blue segments indicate the four possible extensions at each stage

Figure 8 depicts several steps in the algorithm, where the resulting conveyor belt is in red, and the blue segments indicate the possible extensions. Figure 9 shows another example of the final belt constructed in the algorithm where the disks are monotonically separated but not $x$ - and $y$-monotone. 


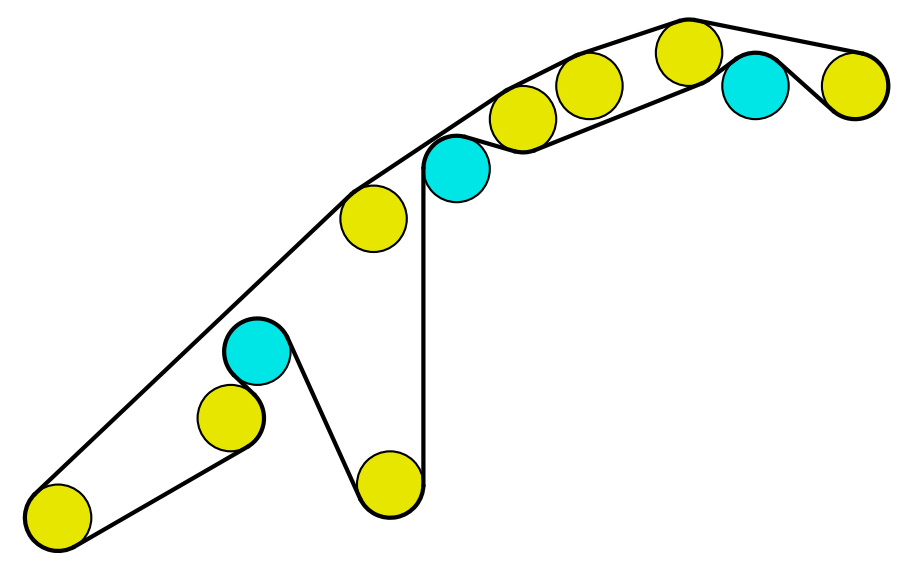

Figure 9: Larger example of the conveyor belt produced by the proof of Theorem 5 .

Theorem 6. The conveyor belt constructed by the algorithm in the proof of Theorem 5 can be constructed in linear time from an input that lists the coordinates of the disk centers in the order of their monotonically separated sequence.

Proof. The upper convex hull can be constructed from this sorted order by a standard Graham scan algorithm, in Andrew's variation using the left-to-right sorted order rather than radial order [3]. In this algorithm, we use a stack data structure to store the sequence of disks in the upper hull of ever-longer prefixes of the input. To extend the prefix, we check if the next disk in the sequence together with the top two disks on the stack form an upper hull that is unchanged if the middle of these three disks is omitted. While this is true we pop the stack, effectively removing the middle disk from consideration. After no more such pops can be performed, we push the new disk onto the stack. At the end of this process, the disks that remain on the stack form the upper hull. Each disk is pushed and popped at most once, so the total time for this part of the algorithm is linear.

To simulate the continuous ray-rotation process by which we generated the partial conveyor belts through the remaining disks, we replace it by a discrete process consisting only of the events at which the continuous process undergoes a discrete change, when the rotating ray reaches a tangency with $D_{i+1}$ or with one of the disks $U_{j}$. There may be many disks $U_{j}$ between $D_{i}$ and $D_{i+1}$; however, by convexity of the sequence of disks $U_{j}$, only two of these disks can cause tangency events: the one that is closest in the sequence to $D_{i}$, and the one that is closest in the sequence to $D_{i+1}$. Therefore, constructing the sequence of discrete events entails only comparing $O(1)$ possible tangencies at each step to determine which of them happens first, and can be performed in constant time per step. Each disk is visited at most once during this process, so the total time for this part of the algorithm is again linear.

A finite sequence of distinct numbers is called bitonic if it has a unique local maximum and a unique local minimum when viewed as a cyclic sequence. Equivalently, it is unimodal up to a cyclic rotation. Since our method constructs conveyor belts from left-to-right, the 

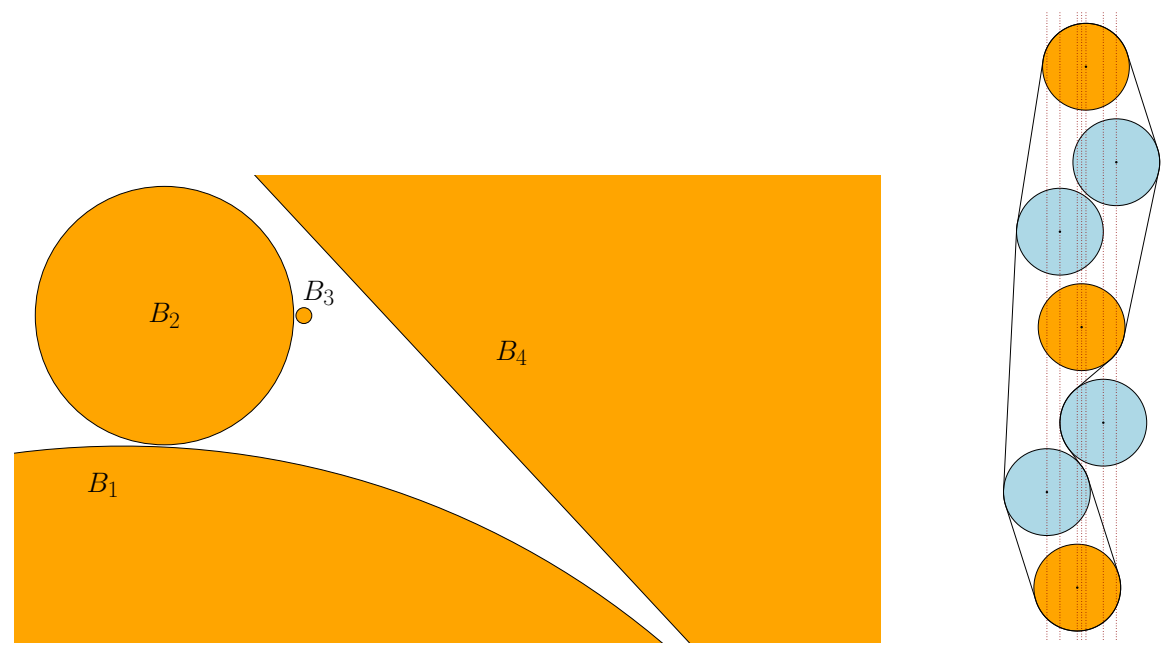

Figure 10: Left: A sequence of $x$ - and $y$-monotone disks with no bitonic conveyor belt. All bitangents between disks $B_{3}$ and $B_{4}$ either pass through $B_{1}$ or $B_{2}$, so 3 must be a local but not global maximum in the sequence of indices of any conveyor belt. Right: A sequence of unit disks with no bitonic conveyor belt. The three orange disks have no unblocked bitangents, and must be separated from each other by at least three local minima or maxima.

result is bitonic with respect to the sequence of $x$-coordinates of disk centers. When a set of disks has a bitonic conveyor belt, it is possible to find such a belt in polynomial time by a straightforward adaptation of the well-known dynamic programming algorithm for bitonic traveling salesperson tours [7]. However, for disks with $x$ - and $y$-monotone centers but arbitrary radii, it is not always possible to find conveyor belts that are bitonic. Figure 10 (left) provides a counterexample. For unit disks that are not monotonically separated, it is also not generally possible to have a tour that is bitonic. In Figure 10 (right) there are three disks that are consecutive in the sorted order by $x$-coordinates, but that have no unblocked bitangent between any two of them. All three disks must be separated from each other in the cyclic sequence of contacts with the conveyor belt, and between any two of them there must be a local minimum or local maximum of the $x$-coordinates.

\section{One-Touch, Arbitrary Radii, and NP-Completeness}

Having solved a particular instance of the conveyor belt problem with unit disks and nonself-intersecting belts, we turn to more general considerations. In this section we focus on the following one-touch conveyor belt problem. We are given as input a collection of disjoint disks in the plane specified by integer center coordinates and arbitrary radii. The goal is to determine whether there exists a conveyor belt that contacts each disk exactly once.

Our proof that this problem is NP-complete will serve as a warm up for the proof that the decision problem for conveyor belts that are not restricted to one touch is also 
NP-complete. Our argument involves the Koebe-Andreev-Thurston circle packing theorem [15] and the notion of maximal planar graphs, so we briefly review these concepts.

Theorem 7 (Koebe-Andreev-Thurston circle packing theorem). For every planar graph $G$ there exists a system of interior-disjoint disks in the plane, corresponding one-to-one with the vertices of $G$, such that two vertices are adjacent in $G$ if and only if the corresponding two disks are tangent.

The centers and radii of these disks are algebraic numbers, but they can be of arbitrarily large degree, so it may not be straightforward to represent them exactly as objects in a symbolic algebra system [5]. Nevertheless, there exist algorithms that can compute their coordinates numerically, to arbitrary precision, in time polynomial in the number of vertices of $G$ and in the number of bits of precision desired $[6,13]$.

A maximal planar graph is a graph embedded in the plane so that all of its faces are triangles, including the unbounded outer face. For maximal planar graphs, the corresponding circle packing is unique up to Möbius transformations of the plane, which preserve circles and their tangencies. In this case, the circle packing can be chosen such that the three vertices of the outer face of the graph are three mutually tangent unit disks, with the rest of the disks all fitting into the triangular region bounded by these three unit disks. For this packing, the disks cannot vary more than exponentially in size: according to the ring lemma for circle packings, there exists a constant $\varepsilon>0$ (not depending on the graph) such that, for any disk $D$ of radius $r$ that touches at most $d$ other disks, the other disks all have radius at least $\varepsilon^{d} r$ [15].

Theorem 8. The one-touch conveyor belt problem is NP-complete.

Proof. We follow the standard outline for a proof that a problem $X$ is NP-complete, by a polynomial-time reduction from a known NP-complete problem $Y$. To do so $X$ and $Y$ must both be decision problems (problems with a YES or NO answer). We must show that $X$ is in NP, and that there exists a polynomial time algorithm for translating all inputs of $Y$ into inputs of $X$ that preserves the answer of each translated input.

To show that the one-touch conveyor belt problem is in NP, we describe a nondeterministic polynomial-time algorithm for it: an algorithm that guesses a solution that can be described in a polynomial number of bits, verifies in deterministic polynomial time that the solution is valid, and if so answers yes. It must be the case that every solvable instance has a solution that causes this algorithm to answer yes, and that no non-solvable instance has such a solution. In our case, the solutions can specify the cyclic order of contacts along the belts and the orientations of each disk, information that can be specified in only $O(n \log n)$ bits for $n$ disks, a polynomial number. If there are $n$ disks, since the belt is one-touch, the resulting belt will have $2 n$ arcs or bitangent segments. The verification algorithm must then test that only consecutive arcs or bitangents intersect, and then only in a single point with compatible directions. It is straightforward to see that these verifications may be performed with $O\left(n^{2}\right)$ tests, so the verification algorithm takes polynomial time, as required. 


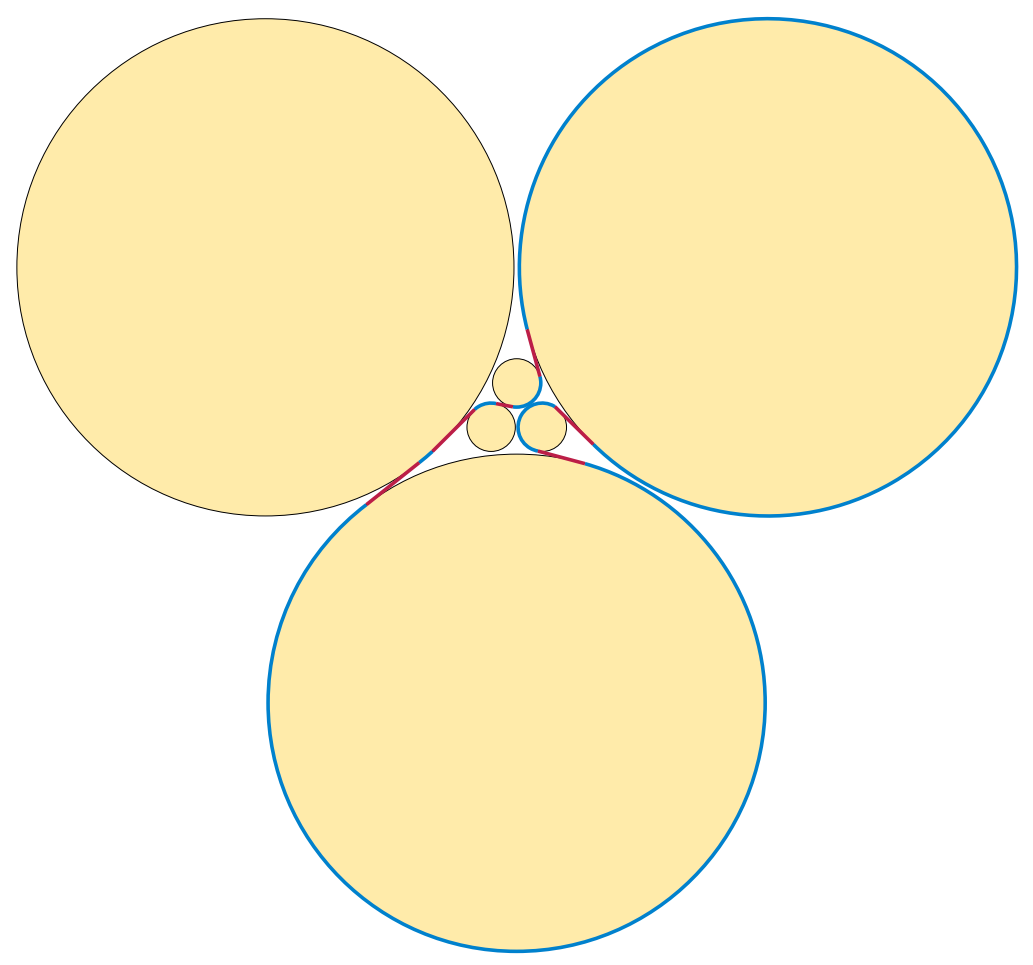

Figure 11: A one-touch conveyor belt for a system of disks constructed by slightly shrinking a circle packing for the graph of the edges and vertices of an octahedron. The belt touches the disks in the order given by a Hamiltonian cycle on the octahedron, and crosses between disks using only inner tangents. Circular arcs on the belt are shown in blue, and bitangents are shown in red.

To prove NP-hardness, we reduce from a known NP-complete problem, determining the existence of a Hamiltonian cycle in a maximal planar graph. This problem was proven NP-complete by Wigderson in 1982 [16]. It is straightforward to modify Wigderson's construction to ensure that the maximal planar graphs that it produces always have an even number of vertices; see Remark 9. This will be useful shortly when we construct a sign-alternating cyclic path, which necessarily must have an even number of vertices.

The reduction begins by representing a given maximal planar graph as a system of touching disks using the circle packing theorem. The only unblocked bitangent segments in the circle packing are outer tangents for the three outermost disks. Shrinking each disk by a factor of $1-\delta$ for $\delta$ sufficiently small will create additional unblocked bitangents only between adjacent disks, see Figure 11. When finding the largest possible $\delta$ for a given triple of disks, we will perform a constant number of polynomial operations and root extractions using the disk radii, so $\delta$ is at least a polynomial in the smallest possible ratio $\varepsilon^{n}$ between tangent disks.

The numerical precision needed to represent the disk centers and their radii accurately enough to perform this shrinkage, and to avoid creating additional bitangencies through numerical inaccuracies, is therefore also at most exponential in the number of disks. 
Numbers with this level of precision may be represented using a linear number of bits, allowing an approximate numerical representation of the circle packing and its shrunken system of disjoint disks to be constructed numerically in polynomial time. By scaling this numerical representation appropriately, we can cause all the disk centers and radii to become integers, as needed for an input to the one-touch conveyor belt problem.

It remains to verify that this transformation from graphs to systems of disks preserves the yes-or-no answers to every input. That is, we must show that a given maximal planar graph $G$ with an even number of vertices has a Hamiltonian cycle if and only if the resulting system of disks has a one-touch conveyor belt. We begin by arguing that, when a one-touch conveyor belt exists, a Hamiltonian cycle also exists. Thus, suppose that we have a one-touch conveyor belt $B$. Since every face of $G$ is a triangle, the only unobstructed bitangents that exist for the disks of the construction are between shrunken disks that, before shrinking, were tangent. Therefore, $B$ can only use these segments to move from one disk to another, and each two consecutive disks along $B$ must be adjacent in $G$. Thus, the cyclic ordering of disks in $B$ corresponds to a cyclic ordering of vertices in $G$ such that each two consecutive vertices in the ordering are adjacent; that is, to a Hamiltonian cycle.

Conversely, suppose we have a Hamiltonian cycle $C$ in $G$; we must show that there exists a one-touch conveyor belt in the corresponding system of shrunken disks. To do so, we use the cyclic ordering of vertices in $C$ as the cyclic order of disks in a belt $B$, and we assign the disks signs that alternate between positive and negative in this cyclic order. This assignment is well-defined since the number of vertices in $G$ is assumed to be even. It corresponds to a curve composed of circular arcs and inner bitangents of pairs of circles that, before shrinking, were tangent. The shrinking process that we perform causes all such inner bitangents to be unobstructed, and disjoint from all the other inner bitangents of other formerly-tangent pairs, so no two such segments can cross, nor can a bitangent segment cross any circular arc. Therefore, the resulting curve is a simple closed curve, composed of arcs and bitangents, with one arc per disk, so it is a one-touch conveyor belt as required.

Figure 11 depicts this construction for the maximal planar graph given by the vertices and edges of a regular octahedron. The one-touch belt shown in the figure connects the six disks shown in the order corresponding to a Hamiltonian cycle through the six vertices of the octahedron.

Remark 9. We now sketch how to modify Wigderson's construction [16] to produce graphs with evenly many vertices. We use the notation of [16]. From Wigderson's graph $K$, one may create a graph $K^{\prime}$ by adding an extra vertex at the midpoint of the edge of $K$ that starts at the central vertex and travels northwest, followed by triangulating the two resulting rectangles by adding two extra edges emanating from the new vertex. In forming Wigderson's graph $M$, use one copy of $K$ and one copy of $K^{\prime}$. The resulting variant of Wigderson's graph $N$ will have 58 instead of 55 vertices, so the graphs $G^{\prime}$ will have a multiple of 58 vertices, an even number. 

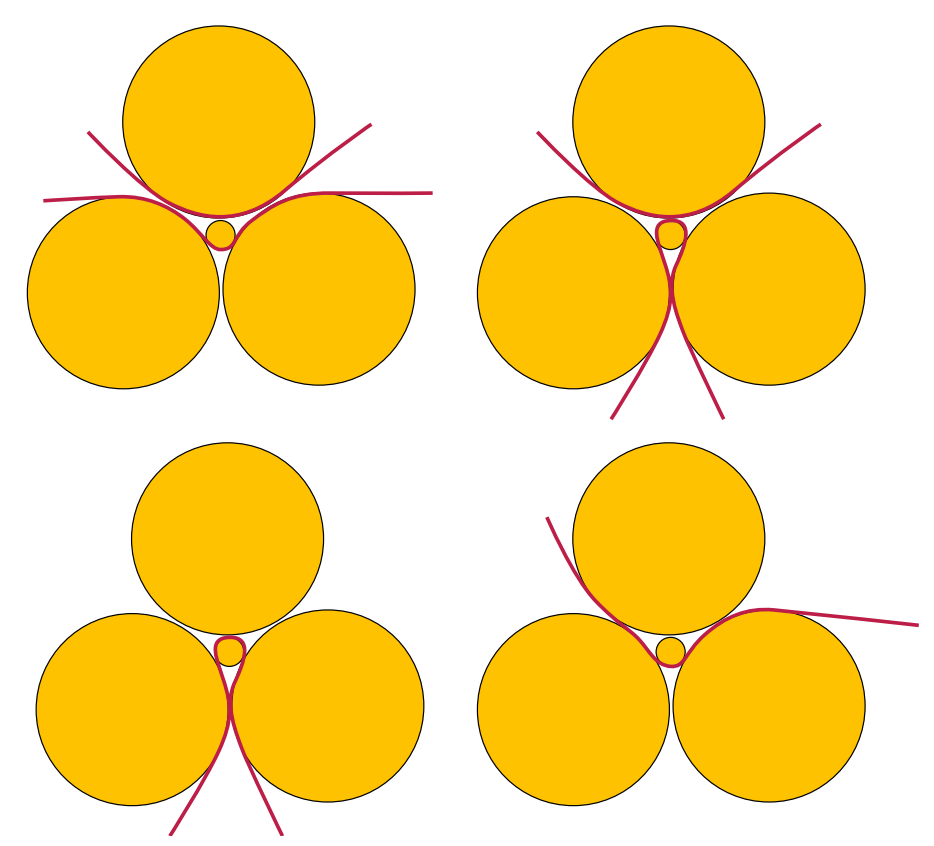

Figure 12: The four possible patterns for a conveyor belt to enter and exit a triangle of a circle packing with a fourth smaller circle inside it: Two double-ply crossings to adjacent triangles (top left), one double-ply crossing (bottom left), a double-ply and two single-ply crossings (top right), or two single-ply crossings (bottom right). The single-ply crossings can be tangent to either of the two circles between which they pass. It is not possible for part of the belt to enter on a double-ply crossing and exit on a single-ply crossing, or vice versa.

Remark 10. Whether counting the number of solutions is \#P-complete remains open. The NP-completeness reduction above is close to parsimonious, but it counts cycles through edges of the outer triangle differently than cycles that do not use those edges. More precisely, one may check that the number of conveyor belts corresponding to a given Hamiltonian cycle is 1 if the cycle involves 0 or 1 edges of the outer triangle, and 2 if the cycle involves 2 edges of the outer triangle.

\section{Multi-Touch, Arbitrary Radii, and NP-Completeness}

Theorem 11. It is NP-complete to determine whether a given system of disks has a conveyor belt, even allowing the belt to touch a single disk multiple times along disjoint arcs.

Proof. As in the one-touch case, we reduce from the Hamiltonian circuit problem, but in a different class of graphs, the cubic 3-connected planar graphs. These graphs are the dual graphs of the maximal planar graphs used for the one-touch problem. Again, testing Hamiltonicity of these graphs is known to be NP-complete [11]; indeed, Wigderson's reduction in [16] is to this older result. 

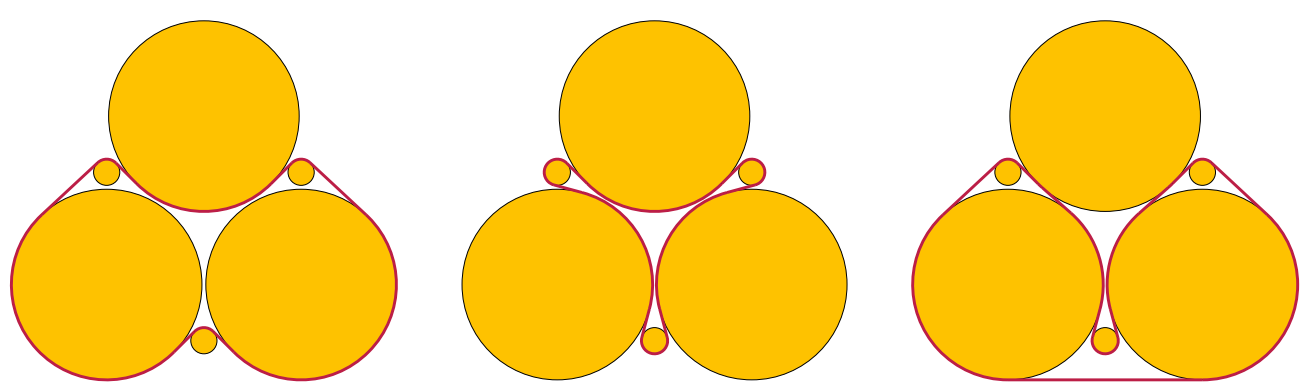

Figure 13: Adding three small disks to the outer triangle of a circle packing restricts a conveyor belt to enter and exit the interior of the packing as shown, up to rotation and reflection.

Our reduction is similar to the one-touch reduction. Let $G$ be a cubic 3-connected planar graph with $n>3$ vertices. Let $G^{\prime}$ be the maximal planar graph dual to $G$. Represent $G^{\prime}$ by a circle packing, and then slightly shrink the disks. This time, we modify the construction by adding another small disk at the radical center of each triple of disks that represent an interior triangle of $G^{\prime}$ (Figure 12), and by adding three more disks tangent to pairs of the outer three disks, small enough that they lie within the convex hull of the outer three disks (Figure 13). The intent of these changes is to force the conveyor belt to visit every face of $G^{\prime}$ and thereby represent a Hamiltonian cycle for the originally given cubic graph $G$, whose vertices correspond to these faces. Since $G$ has $n>3$ vertices, there is at least one interior triple of disks.

For each pair of disks that were initially tangent, a conveyor belt may pass between them at most twice. Call such a crossing single-ply if the conveyor belt passes between those disks once and double-ply if the conveyor belt passes between those disks twice. All of the ways that a belt can enter and exit one of the inner triangles of the circle packing are depicted in Figure 12. By inspecting each case, we find that a belt enters through a single-ply crossing if and only if it exits through a single-ply crossing. It follows that any segment of the conveyor belt passing through the interior of the outer triangle of the circle packing must consist entirely of single-ply or entirely of double-ply crossings.

The disks added for the outside triangle can only be touched by a conveyor belt as shown in Figure 13. We consider each depicted case in turn.

- In Figure 13 (left), the conveyor belt enters and exits the interior of the disk construction via two single-ply connections in regions which correspond to two neighboring triangles of the outer triangular face of $G^{\prime}$. Consequently, if a one-touch conveyor belt exists for the disk construction, it must be single-ply everywhere in the interior as shown in the unique single-ply connection pattern from Figure 12 (lower right). Furthermore, the conveyor belt must enter and exit each region corresponding with a triangle in $G^{\prime}$ exactly once, so it must correspond to a Hamiltonian circuit of $G$. Conversely, it is not hard to see that every Hamiltonian circuit of $G$ can be transformed to a conveyor belt on the constructed disk configuration. Therefore, the disk configuration derived from $G$ has a conveyor belt if and only if $G$ has a Hamiltonian 
circuit.

- In Figure 13 (middle), the conveyor belt enters and exits the interior of the constructed disk configuration via three double-ply connections. The interior connections must then all be double-ply as well, as depicted in Figure 12 (upper left) and (lower left). Furthermore, we can observe from the pictures that both strands enter together from one gap between disks and exit together from another gap between disks. In this sense, double-ply segments form paths. Starting at one of the doubleply connections entering the interior from the outside triangle, the belt must form a path through a sequence of regions corresponding with triangles in $G^{\prime}$. If the path ended at Figure 12 (lower left), there would be a disconnected segment of the belt, so it must end at another connection to the outside triangle. However, the third connection to the outside triangle would then be disconnected from the other two. So, there are no belts of this form.

- In Figure 13 (right), the conveyor belt makes two single-ply connections and one double-ply connection to the interior of the construction. The double-ply connection must pass through the interior to the single-ply connections in order for the belt to be connected, though as noted above the interior connections are all single-ply or all double-ply, so this is not possible and there are again no belts of this form.

Thus, the only possible conveyor belts are ones that makes single-ply connections only, each of which correspond to a Hamiltonian cycle.

\section{Guide Disks}

In this section, we explore the question of how many additional disks must be added in order to guarantee that any disk configuration has a conveyor belt. We show that a linear number of additional disks suffice.

Our construction uses the power diagram for a set of disks in the plane [4]. We briefly recall the definitions here. The power distance from a point $p$ to a disk $D$ with center $c$ and radius $r$ is $|p-c|^{2}-r^{2}$. The power distance is negative if and only if $p$ is inside $D$, and otherwise it is the length of a tangent line from $D$ to $p$. Associated to $D$ is a convex polygon comprised of all points in the plane whose power distance to any disk in the configuration is minimized by its power distance to $D$. The power diagram is the set of these polygons. Since the disks in our configurations are disjoint, $D$ is contained in the interior of its polygon. Geometrically, the power diagram forms a subdivision of the plane into polygons, one per disk, with each disk interior to its polygon. It has $O(n)$ vertices and edges [4, Lemma 1] and can be constructed in time $O(n \log n)[4, \S 5.1]$.

Theorem 12. Any system of $n$ disks can be augmented by $O(n)$ additional disks so that the resulting augmented system of disks has a (one-touch) conveyor belt.

Proof. First construct the power diagram of the disks. The dual graph of the power diagram is the graph whose vertices are polygons in the power diagram, where vertices are connected if their polygons share an edge. Pick a spanning tree of this dual graph. 
Imagine traveling around the "outside" of the spanning tree. This tour corresponds with a cyclic sequence $S$ of vertices and edges of the dual graph. Each vertex in $S$ can be identified with a disk in the collection as constructed from the dual of the power diagram, so we will assume the vertices of $S$ are labeled by the corresponding disks. Conversely, each disk in the collection is represented by some vertex in $S$ since it travels around a spanning tree of the dual of the power diagram. Since the number of edges of the power diagram is linear in $n$, the length of $S$ as a sequence is linear in $n$.

By the definition of the power diagram and dual graph, every pair of consecutive disks $C$ and $D$ in $S$ correspond to adjacent convex polygons in the corresponding subdivision of the plane into polygons. Therefore, there exists at least one point $y$ on their common boundary. If the polygons share an edge, let $y$ be the midpoint of the edge between the polygons of $C$ and $D$ in the power diagram. If the edge is infinite, we may take $y$ to be any point on the interior of the edge. Let $x$ and $z$ be points on the line segments from the centers of $C$ and $D$ to $y$ just outside of $C$ and $D$, respectively. Such points exists since the disks are nonoverlapping. We may represent the edge between $C$ and $D$ in the tree geometrically by a polyline consisting of the two line segments $x y$ and $y z$.

The polylines representing distinct edges in the tree do not cross since the convex polygons in the power diagram form a subdivision of the plane. Taken together, they geometrically represent the spanning tree of the dual graph. By adding small guide disks near each triple $x, y$, and $z$ as needed, we may form a conveyor belt that represents traveling around the "outside" of the spanning tree. Since the length of $S$ is linear in $n$, $O(n)$ guide disks are needed.

In the one-touch model, we may perform a similar construction, but when it would use more than one arc of an input disk we may instead route the belt near the edges of the corresponding power diagram polygon for all but one of these arcs. This will require at most a constant number of disks per edge in the power diagram, so there are again $O(n)$ guide disks in total.

Lemma 13. There exists a configuration $C$ of disks with the property that, whenever $C$ appears as part of a larger configuration $C^{\prime}$, with all disks of $C^{\prime} \backslash C$ disjoint from the convex hull of $C$, every conveyor belt for $C^{\prime}$ must include at least one guide disk interior to the convex hull of $C$.

Proof. Let $G$ be a cubic 3-connected planar graph in which the longest path has fewer than $|V(G)| / 3$ vertices; the existence of $G$ follows from known results on the shortness exponent of cubic 3-connected planar graphs [12]. As in the proof of Theorem 11, represent the dual graph of $G$ by a circle packing (choosing arbitrarily which triple of circles to make exterior), place another smaller tangent circle within each triangle of circles of the packing, and then shrink all of the circles by a small amount to allow a conveyor belt to pass between them without making any additional bitangents available to the belt. Let $C$ be the resulting system of circles.

Then, as in the proof of Theorem 11, any conveyor belt must enter $C$ via at most three single-ply or double-ply crossings between the three pairs of outer circles of $C$. If there 


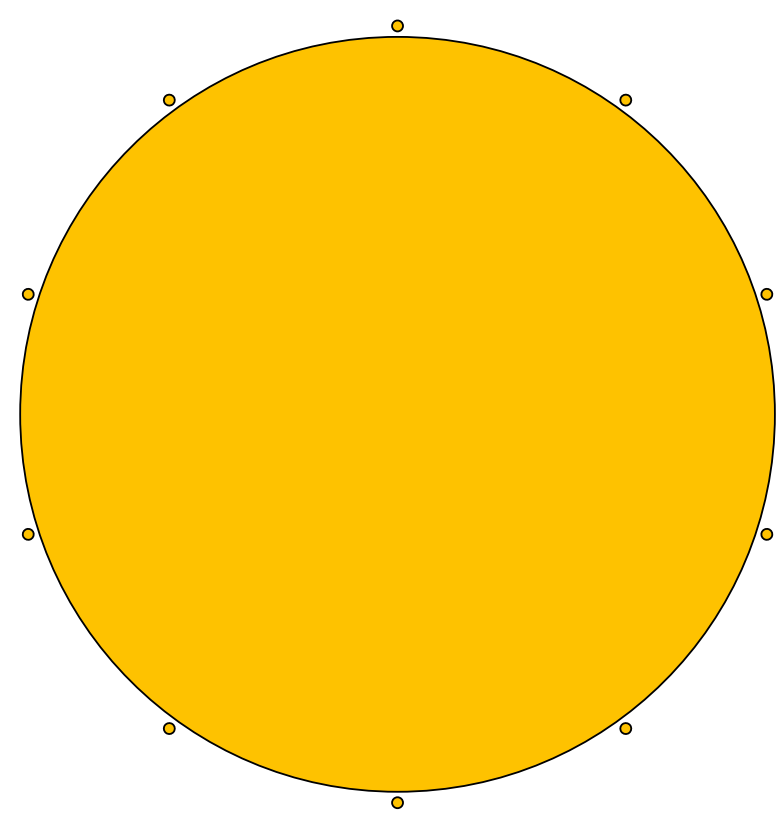

Figure 14: A system of $n$ disks requiring $\Omega(n)$ guide disks for a one-touch conveyor belt

were no guide disks within the convex hull of $C$, the same analysis in Theorem 11 would show that such a belt would necessarily correspond to at most three paths through $G$ that either enter $C$ through one of these crossings and exit through another, or enter $C$ through a double-ply crossing and stop somewhere within $C$. However, because all paths are short, it is not possible for three or fewer paths to touch all vertices of $G$; correspondingly, a conveyor belt without a guide disk within $C$ cannot touch all of the smaller circles within each triangle of circles of $C$. Therefore, at least one guide disk within the convex hull of $C$ is needed.

Theorem 14. In order for one-touch or multi-touch conveyor belts to include $n$ given disks, $\Omega(n)$ guide disks are sometimes necessary.

Proof. For the one-touch case, place $n-1$ small disks at the vertices of a regular polygon, and a large disk at the center of the polygon, in such a way that no two small disks can see each other (Figure 14). Then, in the cyclic sequence of disks given by any one-touch conveyor belt, each of the small disks must be separated from each other by a contact with some other disk, but only one of those contacts can be the large disk. Therefore, there must be at least $n-2$ other guide disks to provide these contacts.

For the multi-touch case, we form a configuration of $\lfloor n /|C|\rfloor$ copies of the configuration $C$ described in Lemma 13, with disjoint convex hulls. Each copy of $C$ requires a guide disk interior to its convex hull, so all the copies together require at least $\lfloor n /|C|\rfloor$ guide disks. 


\section{Future Work}

We conclude with a few questions for further exploration:

1. Can one identify a larger class of unit disks, larger than monotonically separated, which has a conveyor belt? Could one use O'Rouke's hull visibility graph to identify such a beltable collection of unit disks?

2. How many guide disks are necessary to ensure that any traveling salesman tour on the centers of $n$ disks can be a subsequence of a conveyor belt?

3. Is the problem of finding the number of conveyor belts for a given disk configuration \#P-complete?

4. Given $n$ points in the plane, is finding the number of polygons with vertices at the $n$ points also \#P-complete? See [10] for related results and further questions on hardness of counting problem in discrete geometry.

\section{Acknowledgments}

This work was initiated during an open problem session held at the University of Washington in 2016 while the Demaines were Walker-Ames Lecturers, sponsored by the UW Graduate School. We thank Paul Beame, Timea Tihanyi, Ron Irving, and Jamie Walker for their support during the Walker-Ames nomination process; and the 25 students and faculty in math, art, and computer science who attended the session and tackled the problem. We also gratefully acknowledge Joseph O'Rourke and an anonymous referee for detailed comments and helpful suggestions. This work was continued at the 32nd Bellairs Winter Workshop on Computational Geometry, held in 2017 at the Bellairs Research Institute, Barbados. We thank the other participants of that workshop for their input and for creating an exciting atmosphere.

\section{References}

[1] M. Abellanas. Conectando puntos: poligonizaciones y otros problemas relacionados. Gaceta de la Real Sociedad Matematica Española, 11(3):543-558, 2008.

[2] M. Abellanas. Linking geometric objects. In P. Ramos and V. Sacristán, editors, XIV Spanish Meeting on Computational Geometry, In Honor of Ferran Hurtado's 60th Birthday, Alcalá de Henares, June 27-30, 2011, pages 31-32. Centre de Recerca Matemática, Bellaterra, 2011. URL: https://ddd.uab.cat/pub/llibres/2011/ hdl_2072_200199/Documents08_web.pdf.

[3] A. M. Andrew. Another efficient algorithm for convex hulls in two dimensions. Information Processing Letters, 9(5):216-219, 1979. doi:10.1016/0020-0190(79) 90072-3. 
[4] F. Aurenhammer. Power diagrams: properties, algorithms and applications. SIAM J. Comput., 16(1):78-96, 1987. doi:10.1137/0216006.

[5] M. J. Bannister, W. E. Devanny, D. Eppstein, and M. T. Goodrich. The Galois complexity of graph drawing: why numerical solutions are ubiquitous for forcedirected, spectral, and circle packing drawings. J. Graph Algorithms $\&$ Applications, 19(2):619-656, 2015. arXiv:1408.1422, doi:10.7155/jgaa.00349.

[6] C. R. Collins and K. Stephenson. A circle packing algorithm. Comp. Geom., 25(3):233-256, 2003. doi : 10.1016/S0925-7721(02)00099-8.

[7] T. H. Cormen, C. E. Leiserson, R. Rivest, and C. Stein. Introduction to Algorithms. MIT Press, 3rd edition, 2009.

[8] E. D. Demaine and M. L. Demaine. Fun with fonts: algorithmic typography. Theoret. Comput. Sci., 586:111-119, 2015. doi:10.1016/j.tcs.2015.01.054.

[9] E. D. Demaine, M. L. Demaine, and B. Palop. Conveyer-belt alphabet. In H. Aardse and A. van Baalen, editors, Findings in Elasticity, pages 86-89. Pars Foundation, Lars Müller Publishers, April 2010.

[10] D. Eppstein. Counting polygon triangulations is hard. CoRR, abs/1903.04737, 2019. Accepted to SoCG, June 2019. URL: http://arxiv.org/abs/1903.04737.

[11] M. R. Garey, D. S. Johnson, and R. E. Tarjan. The planar Hamiltonian circuit problem is NP-complete. SIAM J. Comput., 5(4):704-714, 1976. doi:10.1137/ 0205049.

[12] B. Grünbaum and H. Walther. Shortness exponents of families of graphs. Journal of Combinatorial Theory. Series A, 14:364-385, 1973. doi:10.1016/0097-3165(73) 90012-5.

[13] B. Mohar. A polynomial time circle packing algorithm. Discrete Math., 117(13):257-263, 1993. doi:10.1016/0012-365X (93)90340-Y.

[14] J. O'Rourke. String-wrapped rotating disks. In A. Márquez, P. Ramos, and J. Urrutia, editors, XIV Spanish Meeting on Computational Geometry, EGC 2011, Dedicated to Ferran Hurtado on the Occasion of His 60th Birthday, Alcalá de Henares, Spain, June 27-30, 2011, Revised Selected Papers, volume 7579 of Lecture Notes in Computer Science, pages 65-78, Cham, 2011. Springer. doi:10.1007/978-3-642-34191-5_6.

[15] K. Stephenson. Introduction to Circle Packing: The Theory of Discrete Analytic Functions. Cambridge University Press, 2005.

[16] A. Wigderson. The complexity of the Hamiltonian circuit problem for maximal planar graphs. Technical Report 298, Princeton University Department of Computer Science, 1982. URL: https://www.math.ias.edu/ avi/PUBLICATIONS/MYPAPERS/ W82a/tech298.pdf. 